Preprint numbers: FTUAM/97-2; NF/DF-01/97

February, 1997

\title{
On the inflationary solutions in higher-derivative gravity with dilaton field
}

\author{
A.L. Maroto ${ }^{a 円}$ and I.L. Shapiro ${ }^{b, c}$ 円 \\ a) Departamento de Física Teórica, Universidad Autónoma de Madrid \\ 28049, Madrid, Spain \\ b) Departamento de Física - ICE, Universidade Federal de Juiz de Fora \\ 33036-330, Juiz de Fora - MG, Brazil. \\ c) Department of Mathematical Analysis, Tomsk Pedagogical University \\ 634041, Tomsk, Russia
}

\begin{abstract}
We discuss the existence of de Sitter inflationary solutions for the string-inspired fourthderivative gravity theories with dilaton field. We consider a space-time of arbitrary dimension $D$ and an arbitrary parametrization of the target space metric. The specific features of the theory in dimension $D=4$ and those of the special ghost-free parametrization of the metric are found. We also consider similar string-inspired theories with torsion and construct an inflationary solution with torsion and dilaton for $D=4$. The stability of the inflationary solutions is also investigated.
\end{abstract}

\section{Introduction}

In the present paper the inflationary cosmological solutions for the $D$-dimensional metric-dilaton theory of gravity with action containing second powers of the curvature tensor are considered. The interest of including into the action terms with higher derivatives [1] is due to the fact that they naturally arise in the string effective action (see, for example, [2]), and also when quantum corrections to the Einstein action are generated by the conformal anomaly of quantized matter fields on curved background [3]. In both cases the effective action contains, along with the metric, an additional scalar field called dilaton. The general fourth derivative metric-dilaton action which interpolates between string-inspired and anomaly-inspired particular actions is too cumbersome [1], and that is why here we restrict our study to the string-inspired higher-derivative models 5 . Such an action doesn't have higher derivatives in the dilaton sector (throughout the paper we use Minkowski signature, notation $R_{\mu \nu \rho}^{\lambda}=\partial_{\rho} \Gamma_{\mu \nu}^{\lambda}-\ldots$ and parametrization of [6]). Let us first consider the torsionless case, then the mentioned effective action can be written as

$$
S_{M}=\frac{2}{\kappa^{2}} \int d^{D} x \sqrt{g} e^{-2 \phi}\left\{-R+4(\partial \phi)^{2}+\alpha^{\prime}\left(a_{1} R_{\lambda \mu \nu \rho} R^{\lambda \mu \nu \rho}+a_{2} R_{\mu \nu} R^{\mu \nu}+a_{3} R^{2}\right)\right\}
$$

\footnotetext{
${ }^{1}$ Electronic address: maroto@eucmax.sim.ucm.es

${ }^{2}$ Electronic address: shapiro@fisica.ufjf.br

${ }^{3}$ One inflationary solution for the anomaly-induced action for the theory with torsion has been obtained in the third reference in [3] (see also [5)
} 
The values of the dimensionless parameters $a_{1}, a_{2}, a_{3}$ have been calculated for bosonic and heterotic string [7] whereas for the superstring the fourth-derivative terms are absent. In fact the coefficients $a_{2}, a_{3}$ derived from string theory contain an arbitrariness related with the possibility of performing the reparametrization of the background metric $g_{\mu \nu}$

$$
g_{\mu \nu} \longrightarrow g_{\mu \nu}^{\prime}=g_{\mu \nu}+\alpha^{\prime}\left(x_{1} R_{\mu \nu}+x_{2} R g_{\mu \nu}\right)+\ldots
$$

where $x_{1,2}$ are arbitrary parameters. In particular in any space-time (target-space) dimension, one can choose some special parametrization in which only the massless graviton field is propagating in the spin-two sector, whereas the massive unphysical ghosts are absent [8, 9]. For such a parametrization the curvature squared terms appear in the combination:

$$
E=R_{\lambda \mu \nu \rho} R^{\lambda \mu \nu \rho}-4 R_{\mu \nu} R^{\mu \nu}+R^{2}
$$

which, in $D=4$, is the integrand of the Gauss-Bonnet topological term. For $D \neq 4$ this term is not topological but it still doesn't contribute to the spin-2 massive pole in the propagator. The propagation of the spin-0 massive states, however, depends on the decomposition of the metric $g_{\mu \nu}$. If one separates the conformal factor of the metric, then the massive spin-0 pole appears. Such a pole, indeed, depends on the gauge fixing and doesn't violate unitarity.

On the other hand, from the string theory point of view such a special parametrization can not be distinguished from the others [10, 11], and that is why it is interesting to explore the nonsingular inflationary solutions for the general action (1). As it was recently demonstrated in [12], the renormalization properties of the effective theory of gravity theory are very different in the special ghost-free parametrization of the metric and in the general one, in which all the ghosts propagate. The two theories are, however, equivalent in the low-energy IR limit [13, 12]. Inflation is another interesting aspect in which the properties of the effective string-inspired gravity may depend (or be independent) on the parametrization. The existence of the inflationary solutions can be introduced in order to constraint the reparametrization arbitrariness in the string effective action, since it would be desirable to have a period of inflation as a natural consequence of string theory at energies below (but not far below) Planck scale. At these energies the first higher derivative corrections to the low-energy string effective action can be relevant.

Recently there has been an extensive study of the (inflationary) cosmological solutions for the string-inspired gravitational theory with higher derivatives restricted by the mentioned ghost-free parametrization of the metric (see [14, 15] and references therein) with $-(1 / 4) a_{2}=a_{3}=a_{1}$. The special case of $a_{1}=a_{2}=0$ was also explored [16]. In the context of the effective field theory for gravity, the cosmological solutions in the dilatonless case obtained from two-loop pure quantum gravity and integrating out conformal free matter in the Standard Model have also been studied [17]. The existence of the de Sitter solutions and the boundary conditions in the presence of the generic higher order terms (but without dilaton or torsion) was discussed in [18] (see also references therein). In the present article we consider the possibility of the inflationary conformally flat solutions in the general theory (11), and therefore start from arbitrary nonrestricted values of $a_{1}, a_{2}, a_{3}$, we also explore the same problem for the theory with torsion.

The paper is organized in the following way. In section 2 the equations of motion for the general theory of the type (1) are derived. In sections 3 and 4 we study the inflationary solutions 
for theories without and with dilaton field. In section 5 the generalization of (1) for the theory with torsion is considered. Section 6 is devoted to the study of the stability of the de Sitter solutions. The last section contains some conclusions.

\section{D-dimensional space-time and general equations of motion}

Consider D-dimensional $k=0$ (conformally flat) Friedmann-Robertson-Walker (FRW) metric:

$$
d s^{2}=-d t^{2}+a(t)^{2} d^{(D-1)} X^{2}
$$

where $d^{(D-1)} X^{2}$ denotes the $(D-1)$-dimensional flat space metric. It is useful to define $b(t)=$ $\log a(t)$ which implies $\dot{b}=\dot{a} / a=H(t)$, with $H$ being the Hubble parameter. Taking into account that in an isotropic and homogeneous space-time the dilaton field can only depend on $t$, we rewrite the action integral (1) as follows:

$$
\begin{gathered}
S_{M}=\int d t e^{(D-1) b} e^{-2 \phi}\left\{(D-1)\left(H^{2} D+2 \dot{H}\right)-4 \dot{\phi}^{2}+\right. \\
+\alpha^{\prime}(D-1)\left[2 a_{1}\left(2 \dot{H}^{2}+4 H^{2} \dot{H}+D H^{4}\right)\right. \\
\left.\left.+a_{2}\left(D \dot{H}^{2}+(D-1) D H^{4}+4(D-1) \dot{H} H^{2}\right)+a_{3}(D-1)\left(H^{2} D+2 \dot{H}\right)^{2}\right]\right\}
\end{gathered}
$$

Since we will deal with different versions of the last action, it is convenient to write the equations of motion for the scale factor and the dilaton field for the generic action of the form:

$$
S=\int d t e^{(D-1) b} e^{-2 \phi} L(H, \dot{H}, \dot{\phi})
$$

They are:

$$
\begin{aligned}
& (D-1) \quad L-(D-1) H \frac{\partial L}{\partial H}+2 \dot{\phi} \frac{\partial L}{\partial H}-\frac{d}{d t} \frac{\partial L}{\partial H}+(D-1)^{2} H^{2} \frac{\partial L}{\partial \dot{H}} \\
& -4 \quad(D-1) \quad H \dot{\phi} \frac{\partial L}{\partial \dot{H}}+2(D-1) H \frac{d}{d t} \frac{\partial L}{\partial \dot{H}}+4 \dot{\phi}^{2} \frac{\partial L}{\partial \dot{H}}-2 \dot{\phi} \frac{d}{d t} \frac{\partial L}{\partial \dot{H}} \\
& +(D-1) \quad \dot{H} \frac{\partial L}{\partial \dot{H}}-2 \ddot{\phi} \frac{\partial L}{\partial \dot{H}}-2 \dot{\phi} \frac{d}{d t} \frac{\partial L}{\partial \dot{H}}+\frac{d^{2}}{d t^{2}} \frac{\partial L}{\partial \dot{H}}=0
\end{aligned}
$$

and

$$
-2 L-(D-1) H \frac{\partial L}{\partial \dot{\phi}}+2 \dot{\phi} \frac{\partial L}{\partial \dot{\phi}}-\frac{d}{d t} \frac{\partial L}{\partial \dot{\phi}}=0
$$

In the next sections the equations (6) and (7) will be applied to the theory (4) with constant and dynamical dilaton field.

\section{Solution without dilaton}

We now return to the low-energy string effective action (四) and consider the simple case with constant dilaton field. Then the action integral can be written as follows:

$$
S_{M}=\int d t e^{(D-1) b}\left\{(D-1)\left(H^{2} D+2 \dot{H}\right)+\alpha^{\prime}\left[\gamma_{1} \dot{H}^{2}+\gamma_{2} H^{2} \dot{H}+\gamma_{3} H^{4}\right]\right\}
$$


where:

$$
\begin{aligned}
\gamma_{1} & =4 a_{1}(D-1)+a_{2} D(D-1)+4 a_{3}(D-1)^{2} \\
\gamma_{2} & =8 a_{1}(D-1)+4 a_{2}(D-1)^{2}+4 a_{3} D(D-1)^{2} \\
\gamma_{3} & =\frac{D}{4} \gamma_{2}
\end{aligned}
$$

The equation of motion for the scale factor reads in this case:

$$
\begin{aligned}
& H^{2}(D-1)^{2}(D-2)+2 \dot{H}(D-1)(D-2)+\alpha^{\prime}\left(3 \dot{H}^{2}(D-1) \gamma_{1}\right. \\
+ & H^{2} \dot{H}\left(2(D-1)^{2} \gamma_{1}+4(D-1) \gamma_{2}-12 \gamma_{3}\right)+H^{4}\left(-3(D-1) \gamma_{3}+(D-1)^{2} \gamma_{2}\right) \\
+ & \left.4 H \ddot{H}(D-1) \gamma_{1}+2 \frac{d}{d t} \ddot{H} \gamma_{1}\right)=0
\end{aligned}
$$

We look for (anti-)de Sitter solutions of the above equation. In terms of the Hubble parameter an (anti-)de Sitter solution is simply $H=H_{0}=$ constant (with our notation de Sitter space-times have constant negative curvature, i.e. $R=-(D-1) D H_{0}^{2}<0$ which implies $H_{0}^{2}>0$, anti-de Sitter space-times have positive curvature), then the above equation reduces to the algebraic one:

$$
H_{0}^{2}(D-1)^{2}(D-2)+\alpha^{\prime} H_{0}^{4} \gamma_{3} \frac{(D-4)(D-1)}{D}=0
$$

The first important point is that this equation does not depend on $\gamma_{1}$ but only on $\gamma_{2,3}$. Such independence has direct sense. One can rewrite the curvature squared terms of the starting action in another basis

$$
\begin{gathered}
a_{1} R_{\lambda \mu \nu \rho}^{2}+a_{2} R_{\mu \nu}^{2}+a_{3} R^{2}= \\
=-\frac{4 a_{1}+(D-2) a_{2}}{4(D-3)} E+\frac{D-2}{D-3}\left(a_{1}+\frac{a_{2}}{4}\right) C^{2}+\frac{\gamma_{1}}{4(D-1)^{2}} R^{2}
\end{gathered}
$$

where

$$
C^{2}=R_{\lambda \mu \nu \rho}^{2}-\frac{4}{D-2} R_{\mu \nu}^{2}+\frac{2}{(D-1)(D-2)} R^{2}
$$

is the square of the Weyl tensor. Indeed for the above FRW metric $C^{2}=0$ and hence only two combinations of the coefficients $a_{1,2,3}$ should be relevant. On the other hand, the $\gamma_{1}$-independence of the equations doesn't indicate that for the given metric the equations are completely independent on the presence of the $R^{2}$ term, because $\gamma_{2,3}$ is not proportional to the coefficient of the GaussBonnet term $E$ in (12). This indicates that the existence of the inflationary de Sitter solutions can, in principle, depend on the choice of parametrization (2) of the "target-space" metric $g_{\mu \nu}$ and also on the space-time dimension. In four dimension not only the $C^{2}$ term is absent, but the GaussBonnet term $E$ contributes as a total derivative to the action (in the absence of the dilaton field) and accordingly only the term proportional to $\gamma_{1} R^{2}$ would contribute to the equations of motion. Since the (anti-)de Sitter solutions are independent on $\gamma_{1}$ this explains that in $D=4$ there is no (anti-)de Sitter solutions different from the trivial Minkowski one $H_{0}=0$. For arbitrary dimension 
the solutions only depend on the combination $\gamma_{3}=2 a_{1} D(D-1)+a_{2}(D-1)^{2} D+a_{3}(D-1)^{2} D^{2}$ and they can be written as:

$$
\begin{aligned}
& H_{0}=0 \\
& H_{0}^{2}=-\frac{D(D-1)(D-2)}{\alpha^{\prime} \gamma_{3}(D-4)}
\end{aligned}
$$

Notice again that four dimensions is the only (apart from $D=2$ ) case in which there is no (anti-)de Sitter solution independently of the parametrization. The sign of $H_{0}^{2}$ (and therefore the type of solution) depends on the sign of $\gamma_{3}$, and thus can be affected by reparametrizations of the target-space metric (2).

\section{Solutions with dilaton}

In the theory with dynamical dilaton the low-energy string effective action (4) can be rewritten in a form similar to $(8)$

$$
S_{M}=\int d t e^{(D-1) b} e^{-2 \phi}\left\{(D-1)\left(H^{2} D+2 \dot{H}\right)-4 \dot{\phi}^{2}+\alpha^{\prime}\left(\gamma_{1} \dot{H}^{2}+\gamma_{2} H^{2} \dot{H}+\gamma_{3} H^{4}\right)\right\}
$$

The corresponding equations of motion for the scale factor and dilaton are:

$$
\begin{aligned}
& H^{2}(D-1)^{2}(D-2)+2 \dot{H}(D-1)(D-2)+\alpha^{\prime}\left(3 \dot{H}^{2}(D-1) \gamma_{1}\right. \\
+ & H^{2} \dot{H}\left(2(D-1)^{2} \gamma_{1}+4(D-1) \gamma_{2}-12 \gamma_{3}\right)+H^{4}\left(-3(D-1) \gamma_{3}+(D-1)^{2} \gamma_{2}\right) \\
+ & \left.4 H \ddot{H}(D-1) \gamma_{1}+2 \frac{d}{d t} \ddot{H} \gamma_{1}\right)-4 \dot{\phi} H(D-1)(D-2)+4 \dot{\phi}^{2}(D-1)-4 \ddot{\phi}(D-1) \\
+ & \alpha^{\prime}\left(\dot{\phi} H \dot{H}\left(-4 \gamma_{2}-8(D-1) \gamma_{1}\right)-8 \frac{(D-2)}{D} \gamma_{3} \dot{\phi} H^{3}+8 \dot{\phi}^{2} \dot{H} \gamma_{1}\right. \\
+ & \left.4 \gamma_{2} \dot{\phi}^{2} H^{2}-8 \dot{\phi} \ddot{H} \gamma_{1}-4 \gamma_{1} \ddot{\phi} \dot{H}-2 \gamma_{2} \ddot{\phi} H^{2}\right)=0
\end{aligned}
$$

and

$$
(D-1)\left(H^{2} D+2 \dot{H}-4 H \dot{\phi}\right)+4 \dot{\phi}^{2}-4 \ddot{\phi}+\alpha^{\prime}\left(\gamma_{1} \dot{H}^{2}+\gamma_{2} H^{2} \dot{H}+\gamma_{3} H^{4}\right)=0
$$

It is easy to see that the above equations may have flat Minkowski space-time as a solution. In this case $H=0$ and then both equations above reduce to the same equation for the dilaton:

$$
-\dot{\phi}^{2}+\ddot{\phi}=0
$$

whose solutions are:

$$
\begin{aligned}
\phi & =\text { constant } \\
\phi & =-\log \left(\frac{t-t_{0}}{\tau}\right)
\end{aligned}
$$

where $t_{0}$ and $\tau$ are integration constants. The $\phi=$ constant case agrees with the Minkowski solution found in the previous section.

Equations (15), (16) are very cumbersome and it is rather difficult to get solutions different from the flat one. As in the previous case, let us try to find the solutions with $H=H_{0}=$ constant, 
that is (anti-)de Sitter space-times. For simplicity we will also assume a linear dilaton, i.e, $\dot{\phi}=v=$ constant. In this case the equations of motion (15) and (16) become algebraic equations for two unknowns:

$$
\begin{aligned}
& (D-1)^{2}(D-2) H_{0}^{2}+\alpha^{\prime} \gamma_{3} \frac{(D-4)(D-1)}{D} H_{0}^{4}-4(D-1)(D-2) v H_{0} \\
& -8 \alpha^{\prime} v \frac{D-2}{D} \gamma_{3} H_{0}^{3}+\frac{16}{D} \alpha^{\prime} v^{2} \gamma_{3} H_{0}^{2}+4(D-1) v^{2}=0
\end{aligned}
$$

and

$$
-4 v^{2}+4 v H_{0}(D-1)-(D-1) D H_{0}^{2}-\alpha^{\prime} \gamma_{3} H_{0}^{4}=0
$$

From these equations we see that the solution can only depend on $\gamma_{3}$, just as in the case without dilaton. The general theorems of algebra ensure that the above higher order system of algebraic equations has some solutions, but it is not explicit that they are real. The analysis of the system shows that there can be three real exact solutions:

i) the trivial one $H=0, v=0$ and

ii) the nontrivial ones

$$
\begin{aligned}
H_{0} & = \pm \sqrt{\frac{1-D}{\alpha^{\prime} \gamma_{3}}} \\
v & =\frac{(D-1)}{2} H_{0}
\end{aligned}
$$

As in the previous case the sign of $H_{0}^{2}$ depends on the sign of $\gamma_{3}$. The above pair of non-trivial solutions are related by the lowest-order scale factor duality [19] given by:

$$
\begin{aligned}
H & \rightarrow-H \\
v & \rightarrow v-(D-1) H
\end{aligned}
$$

Notice also that the presence of a dynamical dilaton modifies the solutions in (13). In addition to this simple solution there are three pairs of complex conjugated solutions. The expression for the complex solutions cannot be given in a compact form for arbitrary dimension, in addition they yield complex values for the dilaton, thus we disregard to present their explicit form here.

\section{Solutions with torsion}

The low-energy string effective action contains in addition to the graviton and dilaton fields an antisymmetric tensor field $H_{\mu \nu \lambda}$, usually referred to as the string torsion. The explicit expression for the effective action in this case is given by [6]:

$$
\begin{aligned}
S_{M} & =\frac{2}{\kappa^{2}} \int d^{D} x \sqrt{g} e^{-2 \phi}\left\{-R+4(\partial \phi)^{2}+\kappa_{0} H_{\alpha \beta \gamma}^{2}+\alpha^{\prime}\left(a_{1} R_{\lambda \mu \nu \rho} R^{\lambda \mu \nu \rho}\right.\right. \\
& +a_{2} R_{\mu \nu} R^{\mu \nu}+a_{3} R^{2}+\kappa_{1} R^{\alpha \beta \rho \sigma} H_{\alpha \beta \lambda} H_{\rho \sigma}{ }^{\lambda} \\
& \left.\left.+\kappa_{2} H_{\mu \nu \lambda} H_{\rho \alpha}^{\nu} H^{\rho \sigma \lambda} H_{\sigma}{ }^{\mu \alpha}+\kappa_{3} H_{\mu \alpha \beta} H_{\nu}{ }^{\alpha \beta} H^{\mu \rho \sigma} H_{\rho \sigma}^{\nu}\right)\right\}
\end{aligned}
$$


Taking into account the antisymmetry of $H_{\alpha \beta \gamma}$, we can write $H_{\alpha \beta \gamma}=\epsilon_{\alpha \beta \gamma \rho} S^{\rho}$. For a FRW space-time and assuming also the homogeneity and isotropy for the $S_{\mu}$ pseudo-vector one has to choose [5] $S_{\mu}=(T(t), 0,0,0)$, then the action (23) reads:

$$
\begin{gathered}
S_{M}=\int d t e^{(D-1) b} e^{-2 \phi}\left\{(D-1)\left(H^{2} D+2 \dot{H}\right)-4 \dot{\phi}^{2}+b_{0} T^{2}\right. \\
+\alpha^{\prime}(D-1)\left[2 a_{1}\left(2 \dot{H}^{2}+4 H^{2} \dot{H}+D H^{4}\right)+\right. \\
+a_{2}\left(D \dot{H}^{2}+(D-1) D H^{4}+4(D-1) \dot{H} H^{2}\right)+a_{3}(D-1)\left(H^{2} D+2 \dot{H}\right)^{2} \\
\left.\left.+b_{1} T^{2} H^{2}+b_{2} T^{4}+b_{3} T^{4}\right]\right\}
\end{gathered}
$$

where

$$
\begin{gathered}
b_{0}=\kappa_{0}(D-1)(D-2)(D-3) \\
b_{1}=-2 \kappa_{1}(D-3)(D-2)(D-1) \\
b_{2}=\kappa_{2}(D-1)(D-2)(D-3)^{2} \\
b_{3}=\kappa_{3}(D-1)(D-2)^{2}(D-3)^{2}
\end{gathered}
$$

The equation of motion for torsion reads:

$$
2 b_{0} T+2 b_{1} \alpha^{\prime} T H^{2}+4 \alpha^{\prime}\left(b_{2}+b_{3}\right) T^{3}=0
$$

and since the action does not contain derivatives of the torsion, it is an algebraic equation. The corresponding solutions are: $T=0$ and

$$
T^{2}=-\frac{b_{0}+b_{1} \alpha^{\prime} H^{2}}{2 \alpha^{\prime}\left(b_{2}+b_{3}\right)}
$$

the first one when substituted back into the action leads to the solutions for metric and dilaton presented in the previous section. The second solution (26) for torsion when substituted gives rise to:

$$
S_{M}=\int d t e^{(D-1) b} e^{-2 \phi}\left\{c_{0}-4 \dot{\phi}^{2}+c_{1} H^{2}+c_{2} \dot{H}+c_{3} H^{2} \dot{H}+c_{4} \dot{H}^{2}+c_{5} H^{4}\right\}
$$

where

$$
\begin{gathered}
c_{0}=-\frac{b_{0}^{2}}{4 \alpha^{\prime}\left(b_{2}+b_{3}\right)}, \quad c_{1}=(D-1) D-\frac{b_{0} b_{1}}{2\left(b_{2}+b_{3}\right)}, \quad c_{2}=2(D-1) \\
c_{3}=\alpha^{\prime} \gamma_{2}, \quad c_{4}=\alpha^{\prime} \gamma_{1}, \quad c_{5}=\alpha^{\prime}\left[\gamma_{3}-\frac{b_{1}^{2}}{4\left(b_{2}+b_{3}\right)}\right]
\end{gathered}
$$

Therefore the effect of a non-vanishing torsion is to modify the coefficients in (团) and introduce a cosmological constant term $c_{0}$. Let us first consider the simple case in which the dilaton is absent. The equation of motion for the scale factor can be obtained directly from (6) and the corresponding (anti-)de Sitter solutions read:

$$
H_{0}^{2}=\frac{A \pm \sqrt{A^{2}-2(D-1)(D-2) B \kappa_{0}^{2}}}{\alpha^{\prime} B}
$$


where: $A=-4(D-1)(D-2)\left(\kappa_{0} \kappa_{1}-\kappa_{2}-(D-2) \kappa_{3}\right)$ and $B=-24 \kappa_{1}^{2}(D-1)(D-2)-8 \kappa_{2} \gamma_{3}(D-$ 4) $/ D-8 \kappa_{3} \gamma_{3}(D-2)(D-4) / D$. A particular case corresponds to the $\sigma$-parametrization in which for the bosonic string

$$
\kappa_{0}=-1 / 12, \quad \kappa_{1}=1 / 8, \quad \kappa_{2}=1 / 96, \quad \kappa_{3}=-1 / 32
$$

while

$$
\gamma_{1}=(D-1), \quad \gamma_{2}=2(D-1), \quad \gamma_{3}=D(D-1) / 2
$$

For these parameters we obtain the following solutions valid for different dimensions.

For $D=2,3$ there is no solution since in that case $T \neq 0$ is not a solution of (25).

For $D=4, \quad \alpha^{\prime} H_{0}^{2}=(4 \pm \sqrt{19}) / 9$. Since one of the above solutions is positive, in this dimension we have inflationary de Sitter solution which appears due to the torsion terms.

For $D=5, \quad \alpha^{\prime} H_{0}^{2}=(42 \pm 4 \sqrt{115}) / 38$, one of the solutions is positive.

For $D=10, \alpha^{\prime} H_{0}^{2}=(-88 \pm 10 \sqrt{77}) / 33$ both solutions are negative.

For $D=26, \alpha^{\prime} H_{0}^{2}=(-840 \pm 2 \sqrt{175727}) / 673$ both solutions are negative.

Thus the existence of the acceptable inflationary solutions for the theory with torsion strongly depends on the dimension of the space-time. In particular such a solution exists for $D=4$ in this parametrization.

In the general case with dynamical dilaton and torsion fields and for an arbitrary parametrization, the equations of motion can again be obtained from (6). The explicit form of the equations is very cumbersome and that is why we present only final results. The corresponding maximally symmetric solutions are the following:

i) Two flat Minkowski solutions with a linear dilaton given by:

$$
\begin{aligned}
H_{0} & =0 \\
v & = \pm \frac{\kappa_{0} \sqrt{(D-1)(D-2)}}{4 \sqrt{\alpha^{\prime} \Lambda}}
\end{aligned}
$$

where $\Lambda=\kappa_{2}+(D-2) \kappa_{3}$. We remark that for the given $k_{2,3}$ this solution (for $v$ ) becomes complex for $D>2$, so we present it here for generality only.

ii) Two pairs of (anti-)de Sitter solutions:

$$
\begin{aligned}
H_{0}^{2} & =\frac{\kappa_{0} \kappa_{1}(D-2)(D-1)+(D-1) \Lambda}{2 \alpha^{\prime}\left(\kappa_{1}^{2}(D-2)(D-1)-\gamma_{3} \Lambda\right)} \\
& \pm \frac{\sqrt{(D-1) \Lambda\left(\gamma_{3} \kappa_{0}^{2}(D-2)+2 \kappa_{0} \kappa_{1}(D-2)(D-1)+(D-1) \Lambda\right)}}{2 \alpha^{\prime}\left(\kappa_{1}^{2}(D-2)(D-1)-\gamma_{3} \Lambda\right)} \\
v & =\frac{(D-1)}{2} H_{0}
\end{aligned}
$$

As in the torsionless case each pair (with opposite signs in $H_{0}$ ) of solutions are related by the duality transformations given in (22). Notice also that these solutions only depend on the $\gamma_{3}$ coefficient (9) of the curvature terms. In the case of the $\sigma$-parametrization for the bosonic string, it can be shown that independently on the dimension only anti-de Sitter solutions arise. 


\section{Stability of the solutions}

In order to study the stability of the above obtained de Sitter solution we will consider small perturbations around them given by:

$$
H(t)=H_{0}+\delta(t), \quad \dot{\phi}(t)=v+\epsilon(t)
$$

One has to notice that while the torsion is taken in the form (24), its perturbations are not relevant. After solving the equations (25) such perturbations reduce to modifications in $\delta(t)$ and $\epsilon(t)$.

Following the general calculational method of section 2, we consider the general theory with action (5) and introduce the notations:

$$
\begin{array}{ccc}
L_{1}=\left.\frac{\partial L}{\partial H}\right|_{0} & L_{2}=\left.\frac{\partial L}{\partial \dot{H}}\right|_{0} & L_{3}=\left.\frac{\partial L}{\partial \dot{\phi}}\right|_{0} \\
L_{11}=\left.\frac{\partial^{2} L}{\partial H^{2}}\right|_{0} & L_{12}=L_{21}=\left.\frac{\partial^{2} L}{\partial H \partial \dot{H}}\right|_{0} & L_{13}=L_{31}=\left.\frac{\partial^{2} L}{\partial H \partial \phi}\right|_{0} \\
L_{22}=\left.\frac{\partial^{2} L}{\partial \dot{H}^{2}}\right|_{0} & L_{23}=L_{32}=\left.\frac{\partial^{2} L}{\partial \dot{H} \partial \dot{\phi}}\right|_{0} & L_{33}=\left.\frac{\partial^{2} L}{\partial \dot{\phi}^{2}}\right|_{0}
\end{array}
$$

where the 0 subindex indicates that the derivatives are taken at the point of extremal $H=$ $H_{0}, \dot{H}=0, \dot{\phi}=v$. Below we only consider real values for $H_{0}$ and $v$.

Linearizing the equations (6) and (7) in the perturbations $\delta$ and $\epsilon$ we obtain the following two equations:

$$
\begin{gathered}
\delta\left[-L_{11} K+L_{12} K^{2}+2(D-1) L_{2} K\right]+\dot{\delta}\left[2(D-1) L_{2}+L_{12} K+L_{22} K^{2}-L_{11}\right]+2 \ddot{\delta} L_{22} K+ \\
+\frac{d}{d t} \ddot{\delta} L_{22}+\epsilon\left[(D-1) L_{3}-L_{13} K+L_{23} K^{2}-4 L_{2} K\right]+\dot{\epsilon}\left[-L_{13}-2 L_{2}-2 L_{23} K\right]+\ddot{\epsilon} L_{23}=0 \\
\delta\left[2 L_{1}+(D-1) L_{3}+L_{13} K\right]+\dot{\delta}\left[2 L_{2}+L_{13}+L_{23} K\right]+\ddot{\delta} L_{23}+\epsilon L_{33} K+\dot{\epsilon} L_{33}=0
\end{gathered}
$$

where $K=(D-1) H_{0}-2 v$. In the simple case without dilaton field, only the first of these equations is needed. This is a third order algebraic equation, and one can analise its solution by standard

methods. In order to simplify this we notice that the equation can be rewritten in the more simple form

$$
\dot{\mathcal{F}}+K \mathcal{F}=0
$$

where $\mathcal{F}=\ddot{\delta} L_{22}+K \dot{\delta} L_{22}+\delta\left(L_{12} K+2 L_{2}(D-1)-L_{11}\right)$ and $K=(D-1) H_{0}$. The characteristic equation of (33) for $\epsilon=0$ is the product of that for the equation $\mathcal{F}=0$ and that for the equation (35). Therefore taking the mode solution $\delta(t)=\exp (\lambda t)$ we arrive at the stability condition ( $\operatorname{Re} \lambda<0$ for all the eigenvalues) in the form:

$$
\begin{aligned}
(D-1) H_{0} & \leq 0 \\
\frac{1}{L_{22}}\left[L_{12}(D-1) H_{0}+2(D-1) L_{2}-L_{11}\right] & >0
\end{aligned}
$$

These conditions are obtained from $\mathcal{F}=0$, for the whole equation (35) we should include the additional condition $\lambda=-K<0$, that is $(D-1) H_{0}>0$ which is not compatible with the first 
condition in (36). Accordingly, all de Sitter solutions are unstable, this property of the de Sitter solutions doesn't depend on the dimension or on the choice of the parametrization.

Returning to the general case with dilaton field, we will consider the non trivial solutions in (21) and (30) which satisfy $K=0$. Then equations (33), (34) reduce to:

$$
\begin{array}{r}
\dot{\delta}\left(-L_{11}+2(D-1) L_{2}\right)+\frac{d}{d t} \ddot{\delta} L_{22}+\epsilon(D-1) L_{3}+\dot{\epsilon}\left(-L_{13}-2 L_{2}\right)+\ddot{\epsilon} L_{23}=0 \\
\delta\left(-2 L_{1}-(D-1) L_{3}\right)+\dot{\delta}\left(-2 L_{2}-L_{13}\right)-\ddot{\delta} L_{23}-\dot{\epsilon} L_{33}=0
\end{array}
$$

As before we consider the mode solutions: $\delta=\exp (\lambda t)$ and $\epsilon=\alpha \exp (\lambda t)$, then the above two equations reduce to a single fourth order algebraic equation. The form of this equation when we especialize to the lagrangian expression in (27) (that also includes the case without torsion (14)) is the following:

$$
\lambda^{4}+\frac{\lambda^{2}}{2 c_{4}}\left(C+2(D-1) B-\frac{B^{2}}{2}\right)+\frac{\lambda}{2 c_{4}}\left(-2 B v(D-1)+\frac{A B}{4}\right)+\frac{v(D-1) A}{2 c_{4}}=0
$$

where:

$$
\begin{gathered}
A=-4 c_{1} H_{0}-8 c_{5} H_{0}^{3}+8(D-1) v \\
B=c_{2}+c_{3} H_{0}^{2} \\
C=-2 c_{1}-12 c_{5} H_{0}^{2}
\end{gathered}
$$

The stability in this case imposes the signs of the coefficients in (38) to be alternate, which implies the following constraints:

$$
\begin{aligned}
\frac{1}{2 c_{4}}\left(C+2(D-1) B-\frac{B^{2}}{2}\right) & >0 \\
\frac{1}{2 c_{4}}\left(-2 B v(D-1)+\frac{A B}{4}\right) & \leq 0 \\
\frac{v(D-1) A}{2 c_{4}} & >0
\end{aligned}
$$

In the particular case without torsion (14), it is possible to get general results from these equations thus: if $\gamma_{1}>0$ and $H_{0} \geq 0$ then the solution is stable, otherwise it is unstable.

The study of stability of the de Sitter solutions with torsion (28) is more involved and it requires to consider the particular parametrizations and dimensions. However, the above three conditions are proportional to $1 / c_{4}$, and $c_{4}$ only depends on $\gamma_{1}$, moreover the dependence on $\gamma_{1}$ only enters through $c_{4}$ and accordingly we can conclude that for a given dimension, the stability of the solutions with dilaton and torsion depends on the parametrization, thus a stable solution for certain $\gamma_{1}$ would be unstable if we change the parametrization to $-\gamma_{1}$. Thus for the given space-time dimension $D$ one can always choose the parametrization in such a way that the de Sitter solutions are unstable.

\section{Conclusion}

We have explored the problem of the inflationary de Sitter solutions in higher derivative stringinspired theories of gravity with dilaton field and with torsion. It is shown that in the absence of 
torsion, the dilaton-free theory may have de Sitter solutions only for $D \neq 4$, whereas the theory with dilaton possesses such a solution (21) with real Hubble parameter for negative $\gamma_{3}$. The lack of (anti-)de Sitter solutions for $D=4$ in the dilatonless theory can be explained by the fact that the crucial Gauss-Bonnet-like term becomes a total divergence in this dimension and doesn't contribute to the equations of motion. When the dilaton field is introduced, $D=4$ doesn't have special features, and in particular there is a real inflationary de Sitter solution with dilaton for $\gamma_{3}<0$.

It is easy to see that the existence of (anti-)de Sitter solutions essentially depends on the parametrization of the metric in the target space. This is because the reparametrization (2) can modify the coefficients $a_{2}$ and $a_{3}$ and therefore change the sign of $\gamma_{3}$ which is essential both for the torsionless case (21) and also in the expressions (28) and (30) for the solutions with torsion.

For the special "ghost-free" parametrization in the theory without torsion, the sign of $\gamma_{3}$ is completely determined by the sign of $a_{1}$ which is (in the Minkowski signature) positive for both bosonic and heterotic string (where $a_{1}=1 / 4,1 / 8$ correspondingly.) [6, 7]. Therefore in this special parametrization there are no inflationary de Sitter solutions, whereas they can exist in other parametrizations. This gives one more illustration to the strong parametrization dependence of the physical properties of the higher order corrections to the string effective action [11].

On the other hand, considering the torsion terms in the action, for non-dynamical dilaton, unstable de Sitter solutions appear for $D=4$. In the general case with dilaton, we obtain the explicit expression for the (anti-)de Sitter solutions.

The investigation of the stability of the (anti-)de Sitter solutions shows that generally this property depends on the particular dimensions and also on the parametrization of the target-space metric. Only in the case of the dilatonless and torsionless theory, one can drow definite conclusion about the universal nonstable nature of the de Sitter solutions. For the torsionless theory with dilaton it proves possible to construct the conditions of stability for the de Sitter solutions explicitly, those do not depend on dimension but only on the parametrization of the metric. In the general case with torsion and dilaton the stability of the de Sitter solutions depends on the parametrization and dimension, but for the given dimension one can always choose parametrization in such a way that the solutions are unstable.

\section{Acknowledgments}

The authors are grateful to the referee for the suggestion to study the stability of the de Sitter solutions, presented in section 6 .

A.L.M. thanks Prof. A. Dobado for useful discussions and also acknowledges partial support by the Ministerio de Educación y Ciencia (Spain) (CICYT AEN96-1634).

I.L.Sh. thanks the Departamento de Fisica - ICE, Universidade Federal de Juiz de Fora for warm hospitality. The work of I.L.Sh. has been supported in part by Russian Foundation for Basic Research under the project No.96-02-16017. 


\section{References}

[1] Stelle K.S.Gen.Rel.Grav.9 (1978) 353; Phys.Rev. 16D (1977) 953.

[2] Green M.B., Schwarz J.H. and Witten E., Superstring Theory (Cambridge University Press, Cambridge, 1987).

[3] Reigert R.Y., Phys.Lett. B134 (1984) 56; Fradkin E.S. and Tseytlin A.A., Phys.Lett. B134 (1984) 187; Buchbinder I.L., Odintsov S.D., Shapiro I.L., Phys.Lett. B162 (1985) 92. Antoniadis I., Mottola E., Phys.Rev. D45 (1992) 2013.

[4] Shapiro I.L., Jacksenaev A.G., Phys. Lett.B324(1994) 284; Elizalde E., Jacksenaev A.G., Odintsov S.D., Shapiro I.L., Phys. Lett.B328(1994) 297.

[5] Buchbinder I.L., Odintsov S.D., Shapiro I.L. Effective Action in Quantum Gravity - IOP Publishing, Bristol and Philadelphia (1992).

[6] Metsaev R.R., Tseytlin A.A., Nucl.Phys. B293 (1987)92.

[7] For the list of references on the higher-loop calculations in the non-linear sigma-models one can see [1] and Ketov S.V., Nonlinear Sigma-models in Quantum Field Theory and Theory of Strings - Nauka, Novosibirsk - (1992, in Russian)

[8] Zwiebach B., Phys.Lett. B156 (1985) 315.

[9] Deser S. and Redlich A.N., Phys.Lett. B176 (1986) 350; Fridling B.E., Jevicki A.,Phys.Lett. B174 (1986) 75; Jones D.R.T., Lowrence A.M., Z.Phys. 42C (1989) 153.

[10] Tseytlin A.A., Phys.Lett. B176 (1986) 92.

[11] Forger K., Ovrut B.A., Theisen S.J. and Waldram D., Higher-Derivative Gravity in String Theory - hep-th/9605145.

[12] Asorey M., López J.L. and Shapiro I.L. Some remarks on high derivative quantum gravity. DFTUZ 96/15, hep-th/9610006

[13] Donoghue J.F., Phys.Rev.Lett. 72 (1994) 2996; Phys.Rev.D50 (1994) 3874.

[14] Boulware D.G. and Deser S., Phys.Rev.Lett. 55 (1985) 2656; Phys. Lett. B175 (1986) 409.

[15] Bento M.C. and Bertolami O., Phys. Lett. B368 (1996) 198.

[16] Whitt B, Phys.Lett. B145 (1984) 176; Barrow J.D. and Ottewill A.C., J.Phys. A16 (1983) 2757; Barrow J.D. and Cotsakis S.,Phys.Lett. B214 (1988) 515; H.-J.Schmidt, Class.Quantum Grav. 6 (1989) 557.

[17] Dobado A. and López A. Phys. Lett.B316(1993) 250; Dobado A. and Maroto A.L.,Phys.Rev. D52 (1995) 1895 
[18] Barrow J.D. and Madsen M., Nucl.Phys. B323 (1989) 242.

[19] Veneziano G., Phys.Lett B265 (1991) 287. 